\title{
Domain Formation in a Fluid Mixed Lipid Bilayer Modulated through Binding of the C2 Protein Motif ${ }^{\dagger}$
}

\author{
Anne Hinderliter, $₫ \S$ Paulo F. F. Almeida," Carl E. Creutz, ${ }^{\ddagger}$ and Rodney L. Biltonen ${ }^{*,+, \perp}$ \\ Departments of Pharmacology and of Biochemistry and Molecular Genetics, University of Virginia Health System, \\ Charlottesville, Virginia 22908, and Departmento de Quimica, Universidade de Coimbra, 3004-535 Coimbra, Portugal
}

Received October 18, 2000; Revised Manuscript Received February 2, 2001

\begin{abstract}
The role and mechanism of formation of lipid domains in a functional membrane have generally received limited attention. Our approach, based on the hypothesis that thermodynamic coupling between lipid-lipid and protein-lipid interactions can lead to domain formation, uses a combination of an experimental lipid bilayer model system and Monte Carlo computer simulations of a simple model of that system. The experimental system is a fluid bilayer composed of a binary mixture of phosphatidylcholine (PC) and phosphatidylserine (PS), containing $4 \%$ of a pyrene-labeled anionic phospholipid. Addition of the $\mathrm{C} 2$ protein motif (a structural domain found in proteins implicated in eukaryotic signal transduction and cellular trafficking processes) to the bilayer first increases and then decreases the excimer/monomer ratio of the pyrene fluorescence. We interpret this to mean that protein binding induces anionic lipid domain formation until the anionic lipid becomes saturated with protein. Monte Carlo simulations were performed on a lattice representing the lipid bilayer to which proteins were added. The important parameters are an unlike lipid-lipid interaction term and an experimentally derived preferential protein-lipid interaction term. The simulations support the experimental conclusion and indicate the existence of a maximum in PS domain size as a function of protein concentration. Thus, lipid-protein coupling is a possible mechanism for both lipid and protein clustering on a fluid bilayer. Such domains could be precursors of larger lipid-protein clusters ('rafts'), which could be important in various biological processes such as signal transduction at the level of the cell membrane.
\end{abstract}

The existence of lipid domains in model and biological membranes has become increasingly accepted as a significant aspect of the current understanding of membrane structure $(1-4)$. The importance of domains in membrane function has been suggested through both experimental and theoretical studies $(5-8)$. Propagation of a signal transduction event usually involves protein-protein interactions, for example, in phosphorylation of proteins or the coupling of receptors to G-protein. Such events will be highly enhanced in magnitude and specificity if the proteins involved are concentrated in the same domain, rather than being distributed over a large number of disconnected domains. Recently, the determination of size and composition of cholesterol/sphingomyelin-rich domains, commonly designated as 'rafts', has received considerable attention (for reviews, see refs 9, 10) with regard to their role in the function of the biological

This work was supported, in part, by Grants GM59205 (R.L.B.) and GM53266 (C.E.C.) from the National Institutes of Health and by Grant praxis/pcna/p/bio/73/96 (PFFA) from the Fundação para a Ciência e Tecnologia, Portugal.

* Address correspondence to this author at the Department of Pharmacology, University of Virginia Health System, P.O. Box 800735 , Charlottesville, VA 22908-0735. Tel.: 804-924-2422; Fax: 804-9820569; E-mail: rlb1t@virginia.edu.

Department of Pharmacology, University of Virginia Health System.

$\S$ Current address: Department of Pharmaceutical Sciences, North Dakota State University, Fargo, ND 58105.

"Departmento de Quimica, Universidade de Coimbra.

${ }^{\perp}$ Department of Biochemistry and Molecular Genetics, University of Virginia Health System. membrane. However, a clear understanding of the physical basis of their formation is yet to be achieved. This is in contrast to gel-fluid phase separation in model lipid bilayers. This phenomenon has been extensively investigated experimentally $(11-14)$ and theoretically $(15-17)$ and is reasonably well understood. However, available evidence suggests that structural or compositional domain coexistence in fluid states, for example, the liquid-ordered and liquid-disordered states $(18-21)$, is probably more relevant for the biological membrane (22).

The nature of lipid domains is normally described in terms of static structures, although some authors have tried to stress the dynamic nature of these domains (9). Furthermore, a perspective focusing on quantitative aspects of their formation is generally lacking. The existence of basically static rafts or domains, albeit freely diffusing in a fluid membrane, would be expected only if the net difference in interactions between raft lipids and non-raft lipids was very large. However, the magnitudes of interactions in biological systems are usually small enough so that processes involving these systems remain reversible. In model bilayers, differences in interaction Gibbs energies between different lipid species are typically of the order of a few hundred calories per mole $(15,17,23-25)$. However, the large number of molecules involved amplifies these interactions, which can lead to domain formation. On the other hand, the changes in Gibbs free energies involved in protein-membrane interactions are typically much larger (several kilocalories per mole of 
protein). These interactions may vary considerably with the lipid composition of the membrane and could lead to formation or disruption of lipid domains. This set of circumstances offers the possibility of fine-tuning in mixed lipid-protein systems. It is precisely this fine-tuning of interactions, which is so prevalent in biological systems, that is considered here.

The approach to this problem combines experimental work with Monte Carlo computer simulations in the study of a protein-lipid bilayer model system composed of two lipid types. The putative lipid domain structure is investigated in the absence and presence of externally added protein. The work is based on the hypothesis that small, cooperative interactions between lipids can be coupled to protein binding, leading to domain formation. Experimentally, we used a fluid bilayer system consisting of large unilamellar vesicles of a binary mixture of phosphatidylcholine (PC) ${ }^{1}$ and phosphatidylserine (PS) containing $4 \mathrm{~mol} \%$ pyrene-labeled anionic phospholipid and a peripheral protein. This peripheral protein is a membrane binding $\mathrm{C} 2$ motif found in a plethora of proteins implicated in eukaryotic signal transduction and cellular trafficking processes.

Protein-induced changes in pyrene excimer/monomer (E/ $\mathrm{M})$ fluorescence of a lipid probe were found to be consistent with protein-induced clustering of the anionic lipid by the C2 domain motif. However, it is not possible to derive domain structure information from the E/M ratio analytically. Therefore, interpretation of the results was approached using Monte Carlo simulations based on a simple model. The main features of this model are the following: (a) The lipid bilayer is represented by a triangular lattice with two types of sites for PS and PC. (b) The protein is represented by a hexagon on the membrane when bound. (c) The binding is fully reversible with the protein preferentially interacting with PS relative to PC. (d) The proteins and lipids are allowed to diffuse in the plane of the membrane. In the simulations, the effect of the magnitudes of the lipid-lipid and proteinlipid interaction free energies on domain sizes is investigated.

To obtain a quantitative description of the system through the Monte Carlo simulations, it is necessary to have an estimate of the Gibbs free energy changes associated with $\mathrm{PC}-$ protein and PS - protein interactions and an interaction parameter describing the difference in the interaction between unlike and like lipids. Thus, the equilibrium binding constants of the $\mathrm{C} 2$ domain to vesicles containing from 0 to $40 \mathrm{~mol} \%$ PS were determined experimentally in the absence of multivalent cations. These binding constants were used to estimate Gibbs free energy changes associated with the protein-membrane interactions. Experimentally it was found that as protein is added to the mixed PS/PC lipid vesicles the $\mathrm{E} / \mathrm{M}$ ratio of pyrene fluorescence goes through a

\footnotetext{
${ }^{1}$ Abbreviations: PC, phosphatidylcholine; PS, phosphatidylserine; POPC, 1-palmitoyl-2-oleoyl-sn-glycero-3-phosphocholine; POPS, 1-palmitoyl-2-oleoyl-sn-glycero-3-phosphoserine; Pyr-PG, 1-hexadecanoyl-2(1-pyrenedecanoyl)-sn-glycero-3-phosphoglycerol; DAG, diacylglycerol; EGTA, ethylene glycol- $O, O^{\prime}$-bis(2-aminoethyl)- $N, N, N^{\prime}, N^{\prime}$-tetraacetic acid; MOPS, 3-morpholinopropanesulfonic acid; MLV, multilamellar vesicles; LUV, large unilamellar vesicles; E/M, pyrene excimer/ monomer ratio; TLC, thin-layer chromatography; RAN, random number; mcc, Monte Carlo cycles; $K_{\mathrm{a}}$, equilibrium association constant; $K_{\mathrm{d}}$, equilibrium dissociation constant; $k_{\text {on }}$, association rate constant; $p_{\text {on }}$, association probability; $p_{\text {off }}$, dissociation probability; $f_{\mathrm{b}}$, fraction of total protein bound.
}

maximum. This appears to correspond to a maximum in the average lipid domain size calculated from the Monte Carlo simulations. The lipid-lipid interaction parameter used in the simulation was varied, and by comparison of the experimentally determined bilayer binding affinities and $\mathrm{E} / \mathrm{M}$ changes with the binding affinity and the E/M ratio deduced from the simulation, the best estimate of the interaction parameter was chosen. The simulations indicate that lipid and protein clustering are highly correlated. This correlated clustering is the result of thermodynamic coupling between the intrinsic tendency for lipid clustering in the absence of any protein-protein interactions and preferential binding of the protein to PS. The type of lipid and protein clusters discussed herein could represent precursors of larger proteinlipid complexes ('rafts'), which could be important in signal transduction at the level of the cell membrane.

\section{MATERIALS AND METHODS}

Materials. 1-Palmitoyl-2-oleoyl-sn-glycero-3-phosphocholine (POPC) and 1-palmitoyl-2-oleoyl-sn-glycero-3-phosphoserine (POPS) were from Avanti Polar Lipids, Inc. (Birmingham, AL). The pyrene-acyl chain labeled phospholipid, 1-hexadecanoyl-2-(1-pyrenedecanoyl)-sn-glycero-3phosphoglycerol, ammonium salt (Pyr-PG), was from Molecular Probes, Inc. (Eugene, OR). All were greater than 99\% pure as determined by thin-layer chromatography (TLC). Hydrated lipid mixtures were periodically extracted for analysis by TLC after fluorescence experiments, and lipid breakdown did not exceed $1-5 \%$. Ethylene glycol- $O, O^{\prime}$-bis(2-aminoethyl)- $N, N, N^{\prime}, N^{\prime}$-tetraacetic acid (EGTA) and potassium chloride were puriss-grade, and 3-morpholinopropanesulfonic acid (MOPS) was Biochemika grade from Fluka Chemical Corp. (Ronkonkoma, NY). Chelex-100 ionexchange resin was from Bio-Rad Labs (Rockville Center, NY). Aprotinin, leupeptin trifluoroacetate, phenylmethylsulfonyl fluoride (PMSF), thrombin, and glutathione agarose beads were from Sigma Chemical Co. (St. Louis, MO). Chloroform, methanol, and benzene were HPLC-grade (Fisher Scientific, Pittsburgh, PA) or Baker Analyzed (J. B. Baker, Inc., Bricktown, NJ). All other chemicals were reagent grade.

Preparation of Solutions. Water was double-distilled through glass. The primary standard phosphate solution in water was prepared from analytical concentrate (J. B. Baker, Inc.). The concentration of phospholipid stock solutions in chloroform was periodically determined by phosphorus analysis (26). All lipid stock solutions were stored in the dark, under an argon atmosphere, at $-72{ }^{\circ} \mathrm{C}$. All buffers used in fluorescence spectroscopy studies were decalcified by passage of the $2 \times$ buffer, $40 \mathrm{mM}$ MOPS, $200 \mathrm{mM} \mathrm{KCl}$, $\mathrm{pH} 7.5$, over a Chelex column before dilution with $\mathrm{dd}_{2} \mathrm{O}$. Terbium chloride hexahydrate was prepared gravimetrically and hydrated in decalcified buffer (Molecular Probes, Inc.). Lyophilized protein was also prepared gravimetrically, and the concentration was confirmed by the Bradford assay (27).

Expression and Purification of Recombinant Proteins. Recombinant proteins were purified by glutathione agarose affinity essentially as documented in Damer and Creutz (28, 29). A pGEX-KG plasmid encoding glutathione $S$-transferase in frame with the $\mathrm{C} 2$ motif nearest the transmembrane sequence of rat synaptotagmin I was transformed into the 
AB1899 strain of Escherichia coli and kindly provided by Dr. Lisa Elferink. The purified C2 motif was desalted on a P-10 G-25 column (Pharmacia LKB Biotechnology, Uppsala, Sweden) equilibrated with or exhaustively dialyzed against $0.5 \mathrm{mM}$ ammonium bicarbonate, $\mathrm{pH} 7.8$, and lyophilized. Lyophilized protein was hydrated in decalcified $2 \mathrm{mM}$ MOPS, 100 mM KCl, pH 7.5.

Preparation of Large Unilamellar Vesicles (LUV). Mixtures of PC and PS were prepared by aliquotting stock solutions of lipid in chloroform into borosilicate culture tubes using gastight syringes (Hamilton Co., Reno, NV). Samples were dried to a thin film under a gentle stream of argon and dried briefly under a vacuum of less than $20 \mathrm{mTorr}$ before being lyophilized from benzene/methanol $(19 / 1, \mathrm{v} / \mathrm{v})$ or left on the vacuum line overnight. The two methods of lipid preparation, thin film or lyophilization, yielded essentially identical results. Lipid was hydrated in the dark above its gel-fluid phase transition temperature with $2 \mathrm{mM}$ MOPS, $100 \mathrm{mM} \mathrm{KCl}, \mathrm{pH} 7.5$, under argon. LUV were prepared by extruding 200 or $250 \mu \mathrm{L}$ of a $4 \mathrm{mM}$ multilamellar vesicle (MLV) dispersion through a $0.1 \mu \mathrm{m}$ pore size polycarbonate filter (Costar Scientific Corp., Cambridge, MA) 30 times using a handheld extruder (Avanti Polar Lipids, Inc., Birmingham, AL). Dynamic light scattering measurements indicated that the average size of the LUV was $120 \mathrm{~nm}$. The dispersion was maintained above its gel-fluid phase transition temperature throughout the extrusion process. LUV containing the fluorescence probe were used within $48 \mathrm{~h}$ of preparation. All other lipid samples were used within a few days. All hydrated lipid samples were stored in the dark under an argon atmosphere at room temperature.

Fluorescence Spectroscopy Experiments. The binding affinity of the $\mathrm{C} 2$ motif for $\mathrm{Tb}^{3+}$ and various lipid mixtures was determined by monitoring changes in energy transfer from tryptophan to bound $\mathrm{Tb}^{3+}$. Advantage is taken of the observation that $\mathrm{Tb}^{3+}$ binds to one or more regions of the protein and that this binding is improved in the presence of lipid. Addition of the cation to a protein solution resulted in a decrease in the protein's emission at $342 \mathrm{~nm}$. This decrease in emission as a function of the cation concentration was used to calculate an apparent dissociation constant of the cation for the protein. Titration of a protein-cation solution with lipid produced a decrease in tryptophan emission and an increase in the cation's emission at $545 \mathrm{~nm}$. These changes as a function of lipid concentration were used to calculate apparent protein-lipid bilayer dissociation constants. These experiments and their analysis are described in detail under Results.

Fluorescence measurements were made on an SLM Aminco 8100 with a Teflon-capped $300 \mu \mathrm{L}$ mini-fluorimeter cell (McCarthy Scientific, Fullerton, CA). The sample was continuously stirred throughout the experiment. In these experiments, the protein's tryptophan was excited at a wavelength of $295 \mathrm{~nm}$. The excitation and emission monochromator band-passes were 1 and $16 \mathrm{~nm}$, respectively, with a gain of 10. Spectra were collected after allowing an incubation time of $15 \mathrm{~min}$ to achieve equilibration. Titration of buffer into the sample did not produce any change in fluorescence.

For experiments employing the fluorescent probe, pyrene, the excitation wavelength was $344 \mathrm{~nm}$, and emission spectra were recorded. Excitation and emission monochromator band-passes were 1 and $8 \mathrm{~nm}$, respectively. Changes in the E/M ratio (emission at $396 \mathrm{~nm} / 470 \mathrm{~nm}$ ) were also examined by collecting $10 \mathrm{~s}$ time traces using dual monochromators. The excimer/monomer (E/M) ratios determined by either data collection method were essentially identical. A gentle stream of argon continuously flowed into the bottom of the fluorimeter cell-holder chamber to minimize $\mathrm{O}_{2}$ quenching. The samples were stirred continuously.

Monte Carlo Computer Simulations. The model used in the simulations consists of a lattice representing the bilayer and an 'aqueous volume' of a size chosen to provide the equivalent total concentrations of lipid $(200 \mu \mathrm{M})$ and protein (variable) used in the E/M experiments. Monte Carlo simulations are performed on a $100 \times 100$ triangular lattice with skew-periodic boundary conditions (30). Each site represents a lipid molecule so that the system has a total of $10^{4}$ lipids. The volume is therefore chosen as $10^{8} / N_{\mathrm{a}}$ liters, where $N_{\mathrm{a}}$ is Avogadro's number. Thus, the concentration of sites is $10^{-4} \mathrm{M}$, representing that half of the lipid in the outer leaflet of a LUV. A fixed number of $\mathrm{C} 2$ proteins is used in each simulation. Equilibration of the protein between the aqueous volume and the lattice is allowed. Thus, the system lattice plus 'volume' is closed, but the lattice alone is an open system with regard to the protein. Each protein, when bound to the bilayer, is represented by a 19 -site hexagon on the lattice. This hexagon is further divided into a core, the inner 7-site hexagon, plus a 12 -site border. The core is responsible for binding to the membrane in general, with an association constant $K_{\mathrm{o}}=\exp \left(-\Delta G_{\mathrm{o}} / R T\right)$, whereas the border preferentially binds negatively charged lipid (PS or pyrenePG) with an additional favorable binding free energy of $\omega_{\mathrm{p}}$ for each site underneath the protein border. The rationale for this protein model is that the $\mathrm{C} 2$ motif domain appears to interact with a membrane surface area of about 20 lipids. This surface includes a patch of hydrophobic residues at the center and four to five basic residues on the surface close to the membrane $(31-33)$. The lattice lipids are either zwitterionic (PC) or negatively charged (PS); some of the latter are 'tagged', representing the pyrene-PG (mole fraction $=$ 0.04), but, apart from this tag, they are completely identical to the other PS lipids. An unlike nearest-neighbor interaction free energy term, $\omega_{\mathrm{ab}}$, describes the differences in the interaction between like and unlike lipids. The allowed moves are protein association with and dissociation from the lattice surface, protein diffusion on the surface (movement of the protein center to a neighboring lattice site), and lipid diffusion (exchange of sites between two lipid neighbors). Proteinprotein overlap is not allowed. Attempted moves in adsorption or diffusion that violate this rule are rejected.

The Metropolis (34) algorithm is used in the simulations. Moves are accepted if the change in free energy associated with the move, $\Delta G$, is less than 0 or if the probability, $p=$ $\exp (-\Delta G / R T)$, is greater than or equal to a selected random number (RAN). The change in free energy is computed from

$$
\Delta G=\omega_{\mathrm{ab}} \Delta n_{\mathrm{ab}}+\Delta P \Delta G_{\mathrm{o}}+\omega_{\mathrm{p}} \Delta\left(n_{\mathrm{b}} P_{\mathrm{b}}\right)
$$

where $\omega_{\mathrm{ab}}=\epsilon_{\mathrm{ab}}-(1 / 2)\left(\epsilon_{\mathrm{aa}}+\epsilon_{\mathrm{bb}}\right)$ is the unlike nearestneighbor interaction parameter, $\Delta n_{\mathrm{ab}}$ is the change in the number of unlike neighbors, $\Delta P$ is the change in the number of bound proteins, $\omega_{\mathrm{p}}$ is the preferential protein-anionic lipid interaction parameter, and $\Delta\left(n_{\mathrm{b}} P_{\mathrm{b}}\right)$ is the change in protein 
border-anionic lipid contacts. $\epsilon_{i j}$ is the intrinsic interaction energy between lipid $i$ and lipid $j$.

The program algorithm is as follows. First, a series of lipid diffusion cycles, typically 50000 Monte Carlo cycles (mcc), are performed in order to achieve equilibrium with respect to lipid distribution (each mcc consists of $10^{4}$ lipid move attempts). Each lipid cycle consists of lipid diffusion with a standard Kawasaki step (30). Second, a series of 50000 total mcc (including protein binding, protein diffusion, and lipid diffusion) are performed in order to achieve equilibrium with respect to protein binding and distribution. Then a series of 100-300 000 total mcc are performed, in the course of which the properties and their averages are calculated. Each of the latter cycles consists of $10^{4}$ operations of protein cycles and lipid cycles. Each protein cycle is of the following type: (1) Pick a random site on the lattice; (2) if there is a protein bound with its center on that site, decide randomly between protein desorption and protein diffusion; (2a) if the choice is protein desorption, calculate the probability of dissociation from

$$
p_{\text {off }}=\left(k_{\text {on }} / K_{\mathrm{a}}\right) /\left(1+k_{\text {on }} / K_{\mathrm{a}}\right)
$$

where $K_{\mathrm{a}}=\exp \left(-\left[\Delta P \Delta G_{\mathrm{o}}+\omega_{\mathrm{p}} \Delta\left(n_{\mathrm{b}} P_{\mathrm{b}}\right)\right] / R T\right)$ and $k_{\text {on }}$ is the on rate constant. The protein is dissociated from the surface if $p_{\text {off }}>$ RAN. (2b) If the choice is protein diffusion, pick a neighbor site randomly and move the protein. Accept the move according to Metropolis criterion and eq 1. (3) If there is no protein bound on the site chosen, and if no other bound protein is close enough to preclude binding because of volume exclusion, try to bind one. Calculate the binding probability from

$$
p_{\mathrm{on}}=[\mathrm{P}] k_{\mathrm{on}} /\left(1+k_{\mathrm{on}} / K_{\mathrm{a}}\right)
$$

where $[\mathrm{P}]$ is the concentration of protein in solution. In eqs 2 and 3, the 1 in the denominator represents the reciprocal of the unit of time used, which is set to the time for a diffusion step on the membrane, approximately $\tau=10^{-7} \mathrm{~s}$. The diffusion limit for $k_{\text {on }}$ is of the order of $10^{11} \mathrm{~s}^{-1} \mathrm{M}^{-1}$ (vesicle), which means that the order of $k_{\mathrm{on}}=1 \tau^{-1} \mathrm{M}^{-1}$. However, because the interest here is only in equilibrium properties, the value of $k_{\text {on }}$ can be chosen quite arbitrarily. To ensure that equilibrium is attained as rapidly as possible, $k_{\text {on }}=10^{3} \tau^{-1} \mathrm{M}^{-1}$ was used to calculate $p_{\text {on }}$.

During the course of a simulation, the number of bound proteins, the distribution of PS domains, and the distribution of protein domains were monitored. A PS domain is defined as a contiguous cluster of PS ( $\geq 2$ anionic lipids). A protein domain is defined as formed by all proteins bound to the same PS domain. If a given bound protein covers sites that belong to two or more PS domains, it is assigned to that domain with the largest number of sites underneath the protein. The number of labeled (tagged PS) contacts, which appears to be proportional to the experimental E/M ratio, was also monitored.

\section{RESULTS}

Monitoring Lipid Domain Formation by Changes in Pyrene Fluorescence. The hypothesis that protein binding can induce lipid domain formation was tested using the first C2 motif of synaptotagmin and a lipid bilayer composed of

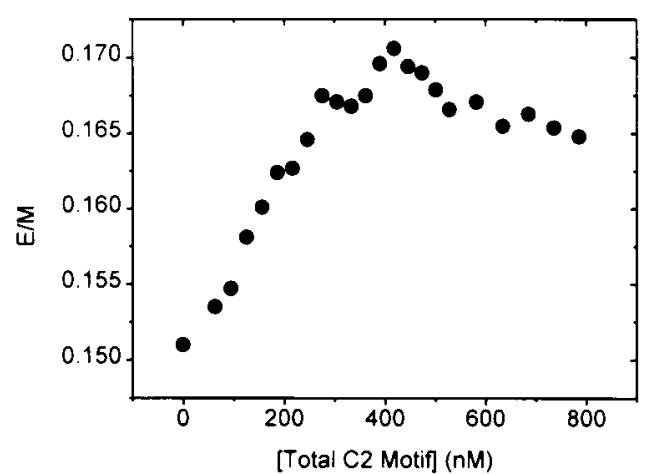

FIGURE 1: Excimer to monomer ratio (E/M) of pyrene-PG in POPC/ POPS LUV as a function of the concentration of $\mathrm{C} 2$ protein motif. The total content of anionic lipid in the LUV is $20 \mathrm{~mol} \%$ (16 mol $\%$ PS plus $4 \mathrm{~mol} \%$ pyrene-PG).

phosphatidylcholine and phosphatidylserine. Assuming that the $\mathrm{C} 2$ motif preferentially associates with PS relative to PC, addition of the protein to the lipid should lead to formation of lipid clusters that are richer in PS relative to the bulk lipid. The experiments were designed such that in the presence of a small amount of anionic lipid essentially all protein was bound to the lipid bilayer. This putative clustering was monitored using pyrene-labeled phosphatidylglycerol (Pyr-PG). Assuming that this lipid mimics the behavior of phosphatidylserine, it likewise should concentrate in the putative PS-rich clusters, leading to an increase in the excimer/monomer fluorescence ratio as described under Materials and Methods. Indeed, when the C2 motif is titrated into a dispersion of LUV of PC/PS/Pyr-PG 80/16/4 (20\% anionic lipid), the behavior observed is shown in Figure 1. Initially, an increase in the E/M ratio is observed, as expected, and at higher concentrations of the protein, the E/M decreases. We interpret this latter effect to mean that when the anionic lipid becomes saturated with protein the clusters begin to dissipate. That is, the anionic lipid will become more randomly dispersed at the highest bound protein to total lipid surface ratios than at lower ratios. This qualitative feature has been discussed previously $(35,36)$.

It could be argued that the increase in E/M is a consequence of an alteration in the diffusion rate rather than altered distribution of pyrene probes. However, given a pyrene lifetime of about 100 ns and a diffusion coefficient in the fluid phase of about $5 \times 10^{-8} \mathrm{~cm}^{2} / \mathrm{s}$, each pyrene probe moves at most 2 lipid diameters before radiative decay. Thus, with only $4 \%$ pyrene probes in a fluid system, no significant effects are expected unless the pyrene moieties are already in close proximity. However, the protein concentration on the bilayer surface is always far from saturating, so the effect of protein crowding on lipid diffusion is probably negligible. Furthermore, the binding of protein to Pyr-PG will, if anything, slow diffusion, resulting in a decrease of E/M, even at small protein concentrations. It thus appears that the clustering effect dominates the process.

C2 Motif Binding to the Lipid Bilayer. Monte Carlo simulations can be used to interpret the E/M results more quantitatively. To do so requires estimates of the intrinsic binding constant of $\mathrm{C} 2$ to pure PC LUV and the Gibbs energy difference associated with the preferential interaction of $\mathrm{C} 2$ with PS lipids relative to that for PC lipids. These estimates can be obtained from experimental evaluation of the association constant of the $\mathrm{C} 2$ motif to lipid bilayers as a 

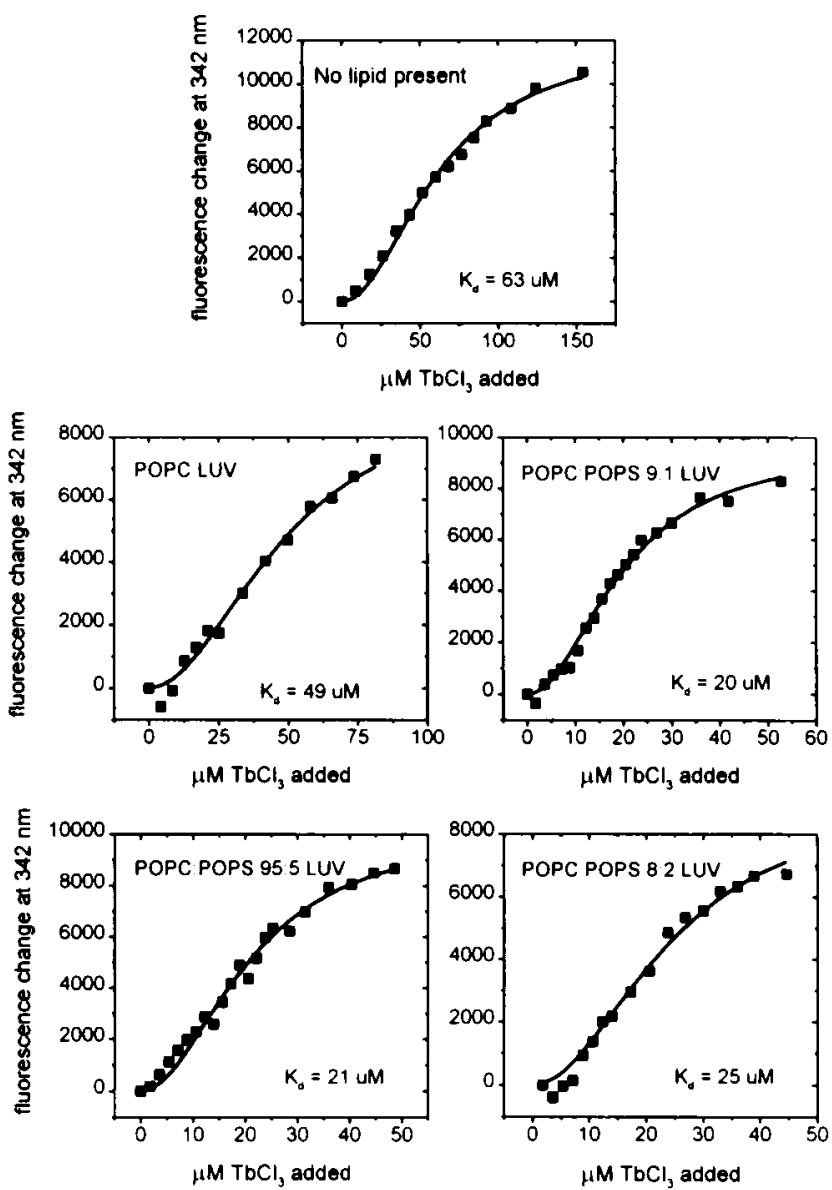

FIGURE 2: Titration of $\mathrm{C} 2$ protein motif with $\mathrm{Tb}^{3+}$ in the absence of lipid (top panel) and in the presence of POPC/POPS LUV with varying PS content (indicated in each panel). In the case of pure POPC, the LUV concentration is $100 \mu \mathrm{M}$ lipid. In the PS-containing LUV, the lipid concentration is such that the total PS concentration is the same in each case: $200 \mu \mathrm{M}$ lipid for $5 \mathrm{~mol} \% \mathrm{PS}, 100 \mu \mathrm{M}$ lipid for $10 \mathrm{~mol} \% \mathrm{PS}, 50 \mu \mathrm{M}$ lipid for $20 \mathrm{~mol} \% \mathrm{PS}$.

function of anionic lipid composition. Previously, changes in protein fluorescence in the presence of $\mathrm{Ca}^{2+}$ were used to obtain estimates of the lipid affinity (36). Those experiments were based upon the observations that $\mathrm{Ca}^{2+}$ binding to $\mathrm{C} 2$ perturbed the fluorescence properties of the protein and that $\mathrm{Ca}^{2+}$ affinity was enhanced in the presence of lipid. However, $\mathrm{Ca}^{2+}$-induced aggregation of the protein and lipid produced large errors in the data. In the present set of experiments, advantage is taken of the observation that $\mathrm{Tb}^{3+}$ binds to one or more regions of the protein and that this binding is improved in the presence of lipid. Thus, energy transfer from the single tryptophan of the $\mathrm{C} 2$ motif to $\mathrm{Tb}^{3+}$ can be used to monitor the interaction between the cation and the protein. This allowed us to take advantage of the larger signal change produced by $\mathrm{Tb}^{3+}$ quenching and perform the experiments at lower lipid concentrations.

The decrease in C2 tryptophan fluorescence as a function of $\mathrm{Tb}^{3+}$ concentration in the absence and presence of lipid is shown in Figure 2. In all cases, the data can be accurately fit to the Hill equation:

$$
\Delta F=\Delta F_{\max }\left(\left[\mathrm{Tb}^{3+}\right] / K_{\mathrm{d}}\right)^{n} /\left(1+\left(\left[\mathrm{Tb}^{3+}\right] / K_{\mathrm{d}}\right)^{n}\right)
$$

$\Delta F_{\max }$ is the maximum decrease in fluorescence achieved under saturating $\left[\mathrm{Tb}^{3+}\right], K_{\mathrm{d}}$ is the apparent dissociation
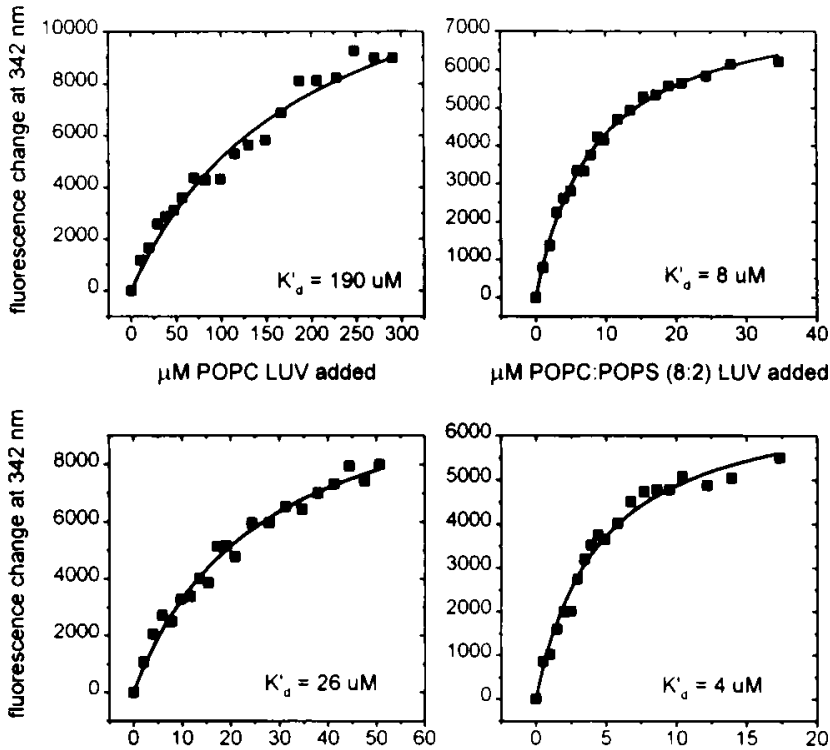

$\mu M$ POPC:POPS (8:2) LUV added
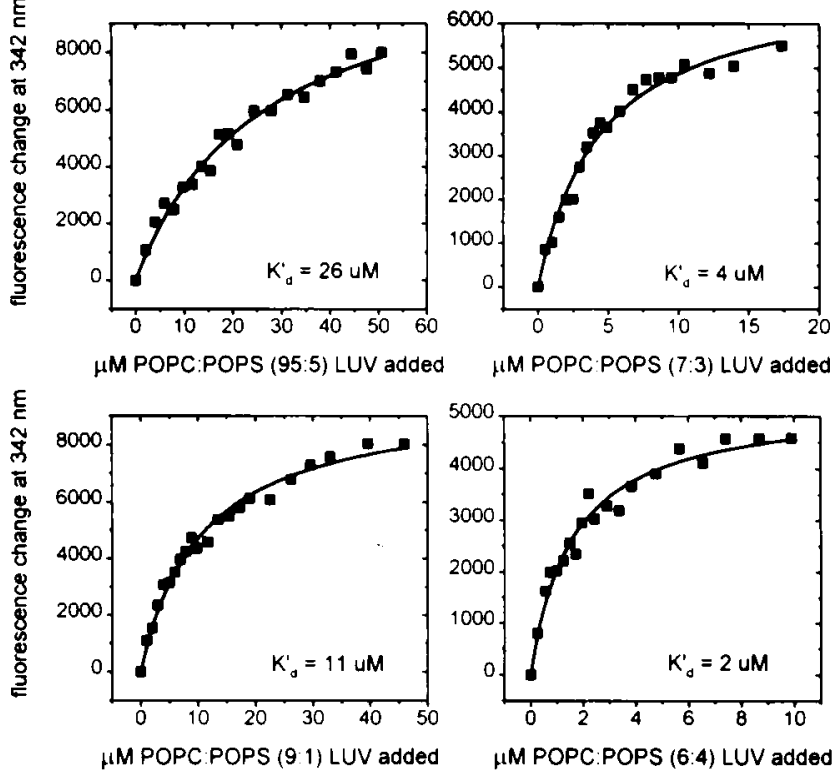

FIGURE 3: Titration of $\mathrm{C} 2$ protein motif with POPC/POPS LUV of varying PS content (indicated in each panel), in the presence of $9.1 \mu \mathrm{M} \mathrm{Tb}{ }^{3+}$. The protein concentration was $388 \mathrm{nM}$, and the fluorescence emission was measured $342 \mathrm{~nm}$.

constant, and $n$ is the Hill coefficient, which was assumed to be constant $(n=2)$ in the analysis of the results presented in Figure 2. A constant value of $n=2$ was assumed in the fitting procedure to minimize the number of variable parameters, but we do not wish to imply that it represents a true stoichiometry for $\mathrm{Tb}^{3+}$ binding. The data shown in Figure 2 indicate that the cation dissociation constant, $K_{\mathrm{d}}$, for the $\mathrm{C} 2$ motif in the absence of lipid is about $65 \mu \mathrm{M}$ and about $20 \mu \mathrm{M}$ in the presence of LUV containing anionic lipid. Except for pure PC LUV, the concentration of lipid used in the experiments is sufficiently high to ensure essentially complete binding of the protein to the vesicles (see Figure 3 ). For pure PC LUV, less than $50 \%$ of the protein is bound to the lipid so that the apparent cation dissociation constant will be an upper estimate of its actual value in the bound state.

The lipid concentration-dependent changes in fluorescence of the $\mathrm{C} 2$ motif in the presence of $9.1 \mu \mathrm{M} \mathrm{Tb} \mathrm{Tb}^{3+}$ are shown in Figure 3 for various binary compositions. All these data can be accurately fit to a rectangular hyperbolic function. The apparent dissociation constants, $K_{\mathrm{d}}^{\prime}$, vary from about $200 \mu \mathrm{M}$ for pure PC to less than $2 \mu \mathrm{M}$ for $6 / 4$ PC/PS vesicles. These apparent values vary by approximately 2 orders of magnitude, which is quantitatively consistent with estimates of the influence of anionic lipid on the binding of pentalysine (37) and phospholipase $\mathrm{A}_{2}$ (38) to binary lipid vesicles containing PC and anionic lipids (PG or PS). These apparent 
Table 1: Dissociation Constants for Protein Binding to POPC/POPS LUV of Varying Composition

\begin{tabular}{cccc}
\hline $\begin{array}{c}\text { LUV } \\
(\text { POPC/POPS })\end{array}$ & $\begin{array}{c}K_{\mathrm{d}}^{\text {app }}(\exp .)^{a} \\
(\mu \mathrm{M})\end{array}$ & $\begin{array}{c}K_{\mathrm{d}}(\exp .)^{b} \\
(\mu \mathrm{M})\end{array}$ & $\begin{array}{c}K_{\mathrm{d}}(\operatorname{sim} .)^{c} \\
(\mu \mathrm{M})\end{array}$ \\
\hline $100 / 0$ & 190 & 190 & 240 \\
$95 / 5$ & 26 & 20 & 45 \\
$90 / 10$ & 11 & 5 & 15 \\
$80 / 20$ & 8 & 2 & 1.5 \\
$70 / 30$ & 4 & 1 & 0.5 \\
$60 / 40$ & 2 & 0.5 & 0.05 \\
\hline
\end{tabular}

${ }^{a}$ Apparent experimental values from Figure 3, in the presence of $9.1 \mu \mathrm{M} \mathrm{Tb}{ }^{3+}$, not corrected for free ligand (lipid) concentration. ${ }^{b}$ Experimental values adjusted to zero $\mathrm{Tb}^{3+}$ using eq 5 and corrected for free ligand (LUV surface) concentration using a C2 motifmembrane contact area of $10-20$ lipids. ${ }^{c}$ Apparent values calculated from the Monte Carlo simulations, using $K_{\mathrm{d}}[$ lipid $]=f_{\mathrm{b}} /\left(1-f_{\mathrm{b}}\right)$, where $f_{\mathrm{b}}$ is the fraction of protein bound to the lattice with 100 proteins in the system.

values can be corrected with eq 5 to yield the dissociation constants, $K_{\mathrm{d}}$, corresponding to binding to the bilayers in the absence of $\mathrm{Tb}^{3+}$ :

$$
\begin{array}{r}
K_{\mathrm{d}}=K_{\mathrm{d}}^{\prime}\left(1+\left(\left[\mathrm{Tb}^{3+}\right] / K_{\mathrm{d}}{ }^{\mathrm{L}}\right)^{2}\right) /\left(1+\left(\left[\mathrm{Tb}^{3+}\right] / K_{\mathrm{d}}^{\mathrm{O}}\right)^{2}\right)= \\
K_{\mathrm{d}}^{\prime}\left(1+(9.1 / 20)^{2}\right) /\left(1+(9.1 / 65)^{2}\right)
\end{array}
$$

where $K_{\mathrm{d}}^{\mathrm{O}}$ and $K_{\mathrm{d}}^{\mathrm{L}}$ are the apparent dissociation constants for $\mathrm{Tb}^{3+}$ binding to the $\mathrm{C} 2$ motif in solution and when bound to the bilayer surface, respectively. Equation 5 can be derived from consideration of the complete thermodynamic cycle of the coupled reactions of $\mathrm{Tb}^{3+}$ and lipid binding to the $\mathrm{C} 2$ motif and using the values for the apparent dissociation constants for $\mathrm{Tb}^{3+}$ binding in the absence and presence of lipid given above. The resulting values of the corrected dissociation constants are given in Table 1. The correction factor included in eq 5 amounts to only about $10 \%$. Therefore, the accuracy of the representation of the $\mathrm{Tb}^{3+}$ binding isotherms in terms of eq 4 is not of great importance in calculating the affinity of the $\mathrm{C} 2$ motif for the lipid bilayers in the absence of cation.

Monte Carlo Computer Simulations. Preliminary simulations were performed to determine the best values of the parameters for the model. In general, the intrinsic tendency for lipid cluster formation is directly proportional to the magnitude of the unlike lipid interaction parameter. If $\omega_{\mathrm{ab}}$ $=0$, then the lipids will distribute randomly. If $\omega_{\mathrm{ab}} \gg 0$, then complete phase separation of the unlike lipids will occur. From the work of Huang et al. (39), it is possible to estimate that the unlike nearest-neighbor interaction parameter between PS and PC is roughly of the order of $200 \mathrm{cal} / \mathrm{mol}$ of lipid, although comparison between their model and the present one is not straightforward. The minimum value corresponding to complete phase separation is a reasonable upper bound. In a pure lipid system in the gel-fluid transition region, a value of $300 \mathrm{cal} / \mathrm{mol}$ of lipid is approximately the minimum value for $\omega_{\mathrm{ab}}$ for the gel-fluid interaction parameter that leads to a first-order phase transition $(17,25)$. It was observed that the affinity of the protein for a bilayer of given composition was only minimally dependent on $\omega_{\mathrm{ab}}$ if it was constrained to the interval 200-320 $\mathrm{cal} / \mathrm{mol}$ of lipid. Furthermore, reasonable agreement was obtained between the binding constants determined experimentally and those calculated from the simulations setting

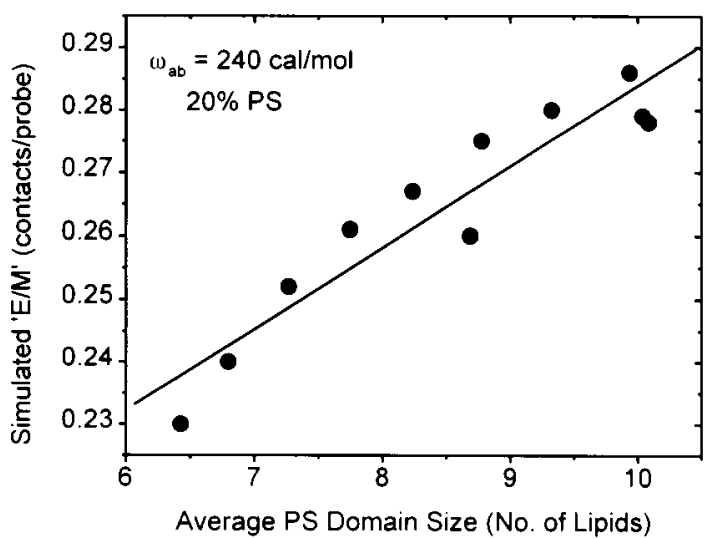

FIGURE 4: Number of contacts between tagged anionic lipids per tagged lipid (4\% of the total lipid) estimated from a Monte Carlo simulation as a function of the average anionic lipid (PS) domain size. The number of tagged lipid contacts provides a theoretical measure of the E/M ratio and is proportional to the PS domain size.
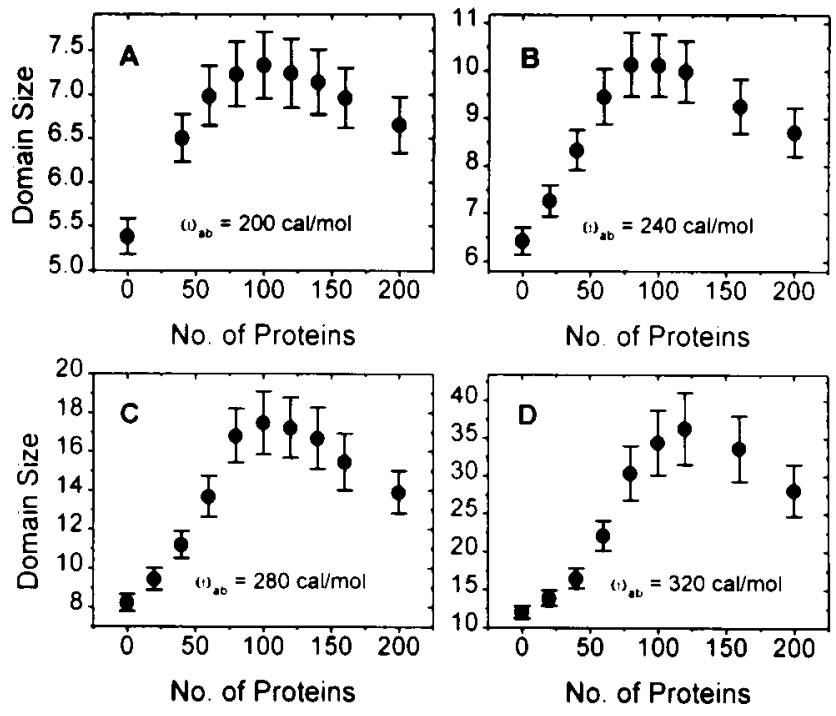

FIGURE 5: Simulations of the effect of the unlike lipid-lipid nearest-neighbor interaction parameter, $\omega_{\mathrm{ab}}$, on the average domain size as a function of protein concentration. PS content was maintained constant at $20 \mathrm{~mol} \%$ PS. (A) $\omega_{\mathrm{ab}}=200 \mathrm{cal} / \mathrm{mol}$, (B) $\omega_{\mathrm{ab}}=240 \mathrm{cal} / \mathrm{mol}$, (C) $\omega_{\mathrm{ab}}=280 \mathrm{cal} / \mathrm{mol}$, (D) $\omega_{\mathrm{ab}}=320 \mathrm{cal} / \mathrm{mol}$. The effect resembles that obtained by varying the PS mol \% at constant $\omega_{\mathrm{ab}}$. Compare with Figure 6, right panels.

$\omega_{\mathrm{p}}=-1 \mathrm{kcal} / \mathrm{mol}$ of lipid and $\Delta G_{\mathrm{o}}=-5 \mathrm{kcal} / \mathrm{mol}$ of protein as shown in Table 1.

The calculated average number of probe pair contacts per probe (tagged PS, $4 \mathrm{~mol} \%$ ) was found to be proportional to the average PS lipid domain size (Figure 4), thus corroborating the hypothesis that the E/M ratio would be a convenient method to assess domain formation experimentally. Two types of Monte Carlo simulations were then performed. In the first set (Figure 5), the value of the unlike nearestneighbor interaction $\omega_{\mathrm{ab}}$ was varied between 200 and 320 $\mathrm{cal} / \mathrm{mol}$ for bilayers containing $20 \mathrm{~mol} \%$ PS. For each $\omega_{\mathrm{ab}}$ value, the total number of proteins was varied between 0 and 200 (corresponding to protein concentrations of $0-2000$ $\mathrm{nM}$ ), most of which were bound to the lattice. The value of $240 \mathrm{cal} / \mathrm{mol}$ results in PS domain sizes as a function of protein concentration that resemble most closely the experimental E/M ratio function shown in Figure 1. In the second 

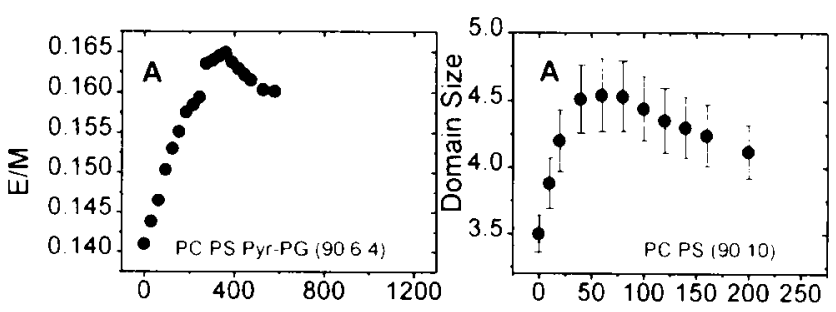

[Total C2 Motif] (nM)
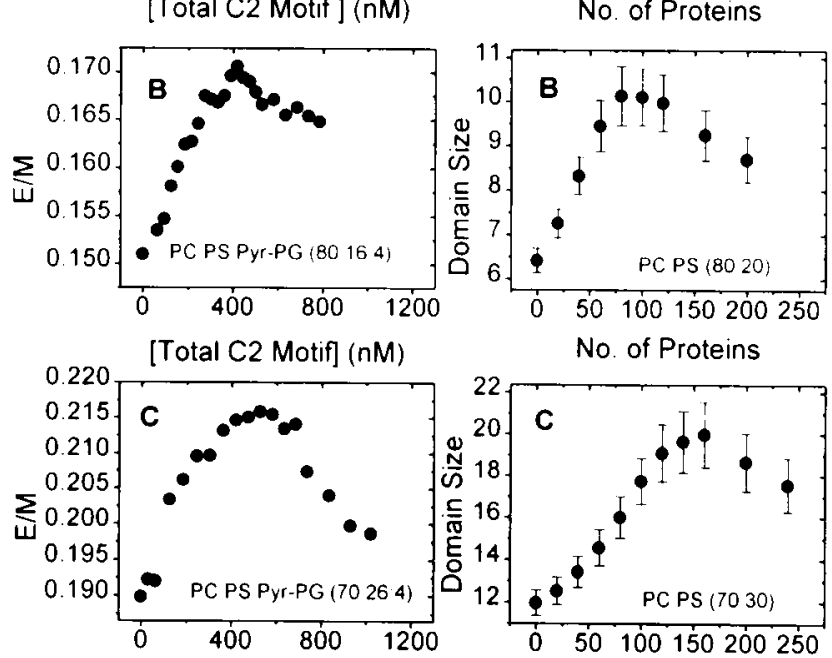

No. of Proteins

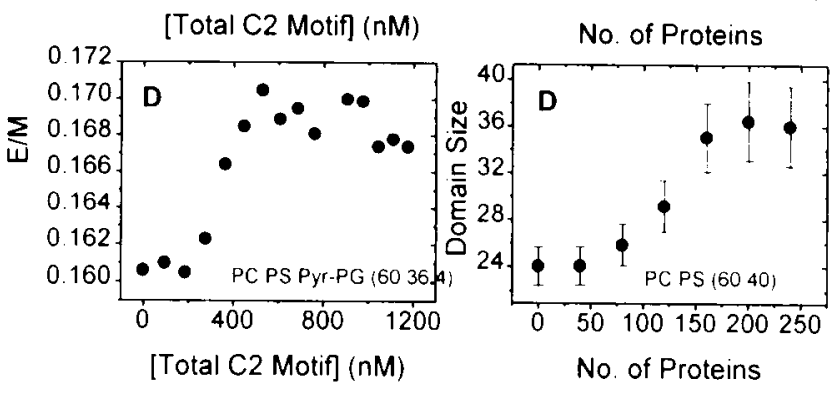

FIGURE 6: Comparison of the experimental E/M ratio (left panels) and average domain size obtained with Monte Carlo simulations (right panels) as a function of protein concentration for various mole percent of the anionic lipid PS: (A) $10 \mathrm{~mol} \%$, (B) $20 \mathrm{~mol}$ $\%$, (C) $30 \mathrm{~mol} \%$, (D) $40 \mathrm{~mol} \%$.

set of simulations, the value of the unlike nearest-neighbor interaction parameter was kept constant at $\omega_{\mathrm{ab}}=240 \mathrm{cal} /$ mol, and the mole fraction of PS was varied between 10 and $40 \%$ (Figure 6, right panels). The general shapes of the experimental functions of $\mathrm{E} / \mathrm{M}$ versus protein concentration (Figure 6, left panels) are quite well reproduced by the simulations. Maxima are observed both in the experimental $\mathrm{E} / \mathrm{M}$ and in the average PS domain size calculated from the simulations at all lipid compositions. Furthermore, a 'lag' in both the E/M and the simulated plots is observed at 40 mol \% anionic lipid.

The existence of protein-lipid (PS) interactions results in significant changes in the equilibrium properties of the membrane, leading to formation of PS-rich domains and protein domains on the lattice surface. The formation of these clusters requires that the unlike lipid interaction parameter, $\omega_{\mathrm{ab}},>0$ and the protein-lipid preferential interaction parameter, $\omega_{\mathrm{p}}, \ll 0$. For example, if $\omega_{\mathrm{ab}}=0$ and $\omega_{\mathrm{p}}=-1$ $\mathrm{kcal} / \mathrm{mol}$, the binding of the 80 proteins to the lattice produces an increase in the average lipid cluster size from 3.4 to 3.7 lipids when the fraction of anionic lipid is $20 \%$. This is to be compared to an increase from 6.4 to 10.2 lipids (Figure
$6 \mathrm{~B}$, right) when $\omega_{\mathrm{ab}}=240 \mathrm{cal} / \mathrm{mol}$. Similarly, if $\omega_{\mathrm{p}}=0$, protein binding has no influence on the lipid distribution. This is demonstrated in the snapshot of a lipid distribution shown in Figure 7 (upper left panel) simulated for $\omega_{\mathrm{ab}}=$ $240 \mathrm{cal} / \mathrm{mol}$. This snapshot is typical for the lipid distribution with either 0 or 80 proteins bound to the lattice if $\omega_{\mathrm{p}}=0$. The protein distribution corresponding to Figure 7 (upper left panel) is shown in Figure 7 (upper right panel), where, at most, a few protein domains exist, none larger than 3 proteins in size. However, if $\omega_{\mathrm{p}}=-1 \mathrm{kcal} / \mathrm{mol}$, binding of 80 proteins to the lattice induces a major change in the lipid distribution as demonstrated by the snapshot shown in Figure 7 (lower left panel) where several large clusters of anionic lipid can be observed. The corresponding snapshot of the protein distribution is shown in Figure 7 (lower right panel). Here we observe significant protein clustering including the existence of one very large cluster of proteins. In general, the clustering of proteins and anionic lipids are in 1:1 correspondence as demonstrated by the linear relationship between protein cluster size and average lipid domain size shown in Figure 8. Although no attractive protein-protein interaction was introduced into the model to quantitatively rationalize the experimental results, large protein domains can be formed in a process mediated by only preferential protein-lipid and lipid-lipid interactions.

A typical lipid cluster distribution function representing the average number of PS lipids found in clusters of a prescribed cluster size is shown in Figure 9 for a lattice with 100 proteins bound (solid line) and no proteins bound (dashed line). The effect of protein binding is evident in a decrease of the fraction of PS lipids in small clusters with a concomitant increase in the fraction in very large clusters. Clusters of about 20 lipids, the number of sites under a bound protein, are especially favored, as seen by the shoulder in the cluster distribution function in the presence of protein. In Figure 10 the protein cluster distribution function is shown in terms of the average number of proteins found in clusters of a given cluster size for these two cases. For the case with no preferential protein-lipid interaction, we obtain the expected exponential decay as a function of the cluster size (note the logarithmic scale in Figure 10) whereas a nonexponential decay in the function is observed when $\omega_{\mathrm{p}}<0$. In a previous publication [Hinderliter et al. (36)], we showed that the distribution function of lipid and protein clusters could be extremely structured, corresponding to domains of the $1,2,3, \ldots$ proteins per cluster. Those preliminary simulations were performed assuming 25 preferential anionic binding sites per protein and a much larger intrinsic propensity for the lipids to cluster than in the current case. Comparison of the current results with the previous results demonstrates that the distribution of lipid and protein clusters is a strong function of the quantitative details of the interactions in the model. However, the basic result from both studies is the same. Thermodynamic coupling of preferential protein-lipid interactions with lipid-lipid interactions can lead to extensive protein and lipid clustering, even in the absence of specific protein-protein interactions.

\section{DISCUSSION}

We have tested the hypothesis that cooperative interactions between lipids thermodynamically coupled to protein binding can lead to lipid and protein clustering. This study examined 

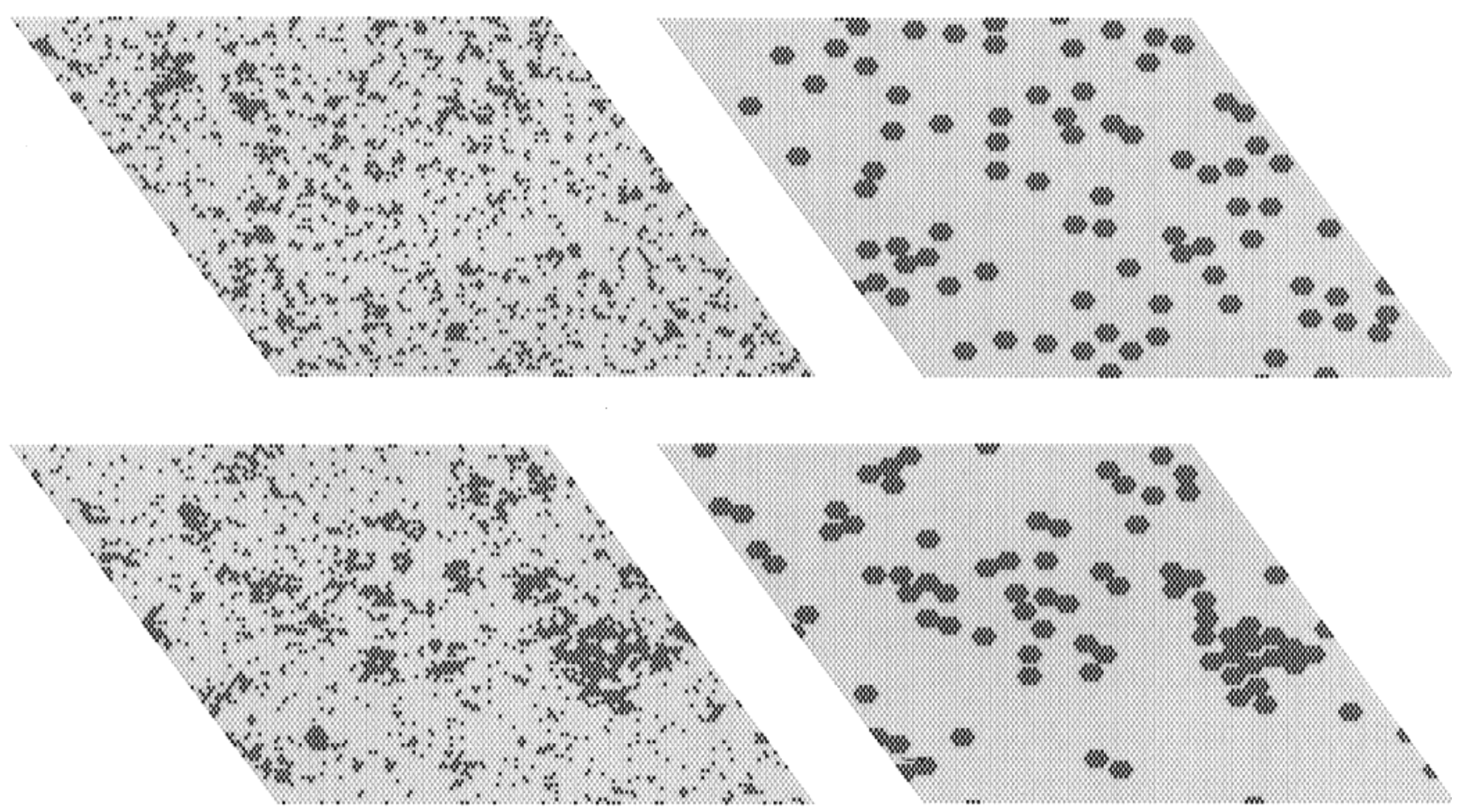

FIGURE 7: Snapshots of the lipid lattice $(100 \times 100$ sites $)$. (Upper left panel) A lipid distribution with no proteins present. Darker sites represent PS lipids. (Upper right panel) A random protein distribution for this system with 80 proteins, but with no preferential protein-PS interaction. Each dark hexagon on the lattice represents a protein. (Lower left panel) A lipid distribution with 80 proteins present and a protein-PS preferential interaction of $-1 \mathrm{kcal} / \mathrm{mol}$. (Lower right panel) A protein distribution for 80 proteins with a preferential proteinPS interaction of $-1 \mathrm{kcal} / \mathrm{mol}$. This panel is the protein counterpart of the lipid snapshot in the lower left panel. In all cases, $\omega_{\mathrm{ab}}=240$ $\mathrm{cal} / \mathrm{mol}$.

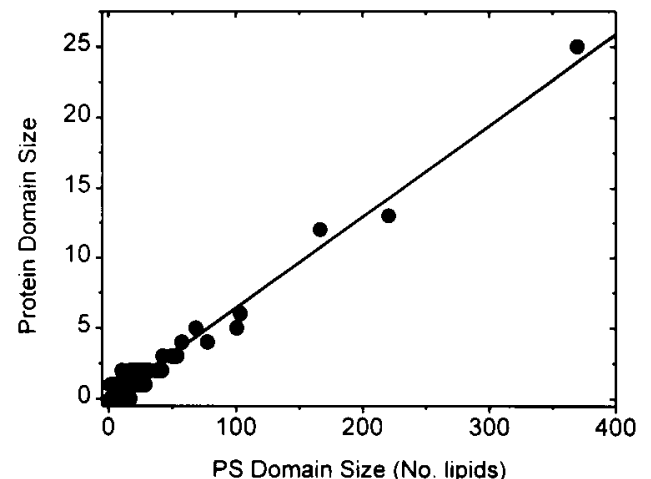

FIGURE 8: Protein domain size as a function of lipid (PS) domain size obtained for Monte Carlo simulations. The linearity is a manifestation of the coupling between protein-lipid and lipidlipid interactions.

the induction of membrane lipid domains enriched in anionic lipid upon the binding of a peripheral membrane protein with a basic face. The $\mathrm{C} 2$ motif from rat synaptotagmin I was selected for this study because of its homology to the membrane binding motif in PKC and for its conserved, abundant occurrence in a wide variety of proteins involved in signal transduction and membrane trafficking events. The model membrane system used was a fluid state, binary lipid mixture comprised of phosphatidylserine and phosphatidylcholine.

An anionic phospholipid labeled with a pyrene-labeled acyl chain was included in the lipid mixture to experimentally monitor changes in the local concentration of the anionic lipid. Increases in the pyrene excimer/monomer fluorescence ratio with protein addition were initially observed (Figure 6 , left). This is interpreted to mean that the concentration of

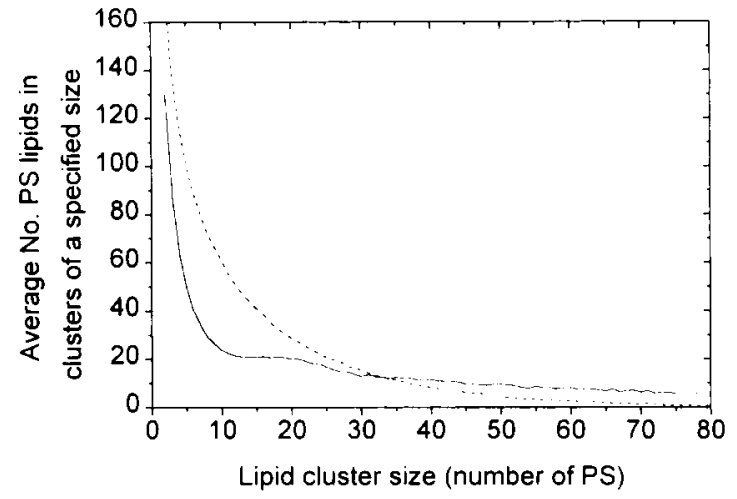

FIGURE 9: Lipid cluster distribution functions in the absence and presence of bound $\mathrm{C} 2$ motif protein. Both curves refer to a PC/PS $80 / 20$ mixture with an interaction parameter of $\omega_{\mathrm{ab}}=240 \mathrm{cal} / \mathrm{mol}$ of lipid. The curves represent the average number of PS lipids found in clusters with a specified number of PS lipids in a $100 \times 100$ lattice. The dashed curve is the distribution in the absence of proteins and the solid curve with 100 proteins in the system where $\omega_{\mathrm{p}}=-1 \mathrm{kcal} / \mathrm{mol}$ (essentially all proteins are bound). These average values were calculated by averaging over several thousand snapshots, many of which would not have clusters of a specified size. Therefore, the average value can be less than the cluster size (e.g., very large clusters).

the pyrene-labeled and other anionic lipids in the neighborhood of the protein increases as a consequence of their favorable interaction with the protein. This, in turn, increases the probability of existence of two neighboring pyrene moieties that can form excimers. Additional binding of protein leads to a broad maximum in the $\mathrm{E} / \mathrm{M}$ ratio, indicating that the anionic lipid is becoming saturated with protein. Further addition of protein causes a reduction in the 


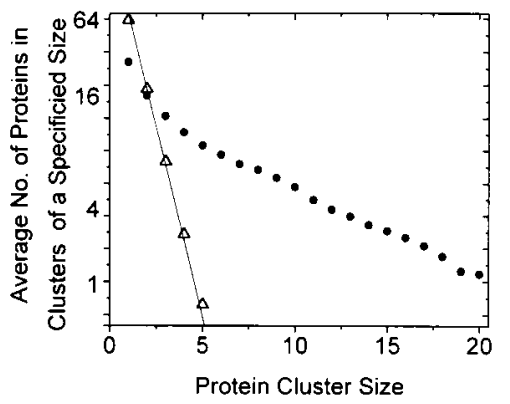

FIGURE 10: Protein cluster distribution functions without preferential protein-PS interaction (triangles) and with a $-1 \mathrm{kcal} / \mathrm{mol}$ protein-PS preferential interaction (circles). The average number of proteins bound to the lattice is the same in both cases ( 80 proteins in the system, of which essentially all are bound). These distributions represent the average number of proteins found in a protein cluster of a given size. Note that the ordinate is logarithmic. The straight line represents an exponential decay of the cluster frequency with cluster size, as expected for a random system. These average values are calculated by averaging over several thousand snapshots, many of which will not have clusters of a specified size. Therefore, the average value can be less than the cluster size.

experimental $\mathrm{E} / \mathrm{M}$ ratio, indicating dissipation of the clusters as the fixed number of pyrene-labeled and other anionic phospholipids become dispersed among the greater number of bound proteins.

The above interpretation of the experimental results is supported by results of Monte Carlo computer simulations based on the simple model described under Materials and Methods. The Monte Carlo simulations show that anionic lipid domains initially increase in size upon protein binding, reach a maximum, and then become smaller (Figure 6, right). The average anionic lipid domain size was found to be linearly related to the number of contacts between tagged lipid sites (representing the pyrene probes), which validates the interpretation of experimental $\mathrm{E} / \mathrm{M}$ ratio as a parameter indicative of domain formation (Figure 4). Lipid domain formation and protein domain formation are coupled through the preferential interaction of the $\mathrm{C} 2$ domain with the anionic lipid, which is most easily seen by the linear relation between lipid domain size and protein domain size shown in Figure 8. The cluster size distribution in the presence of protein is highly asymmetric with a few very large domains comprised of 100-400 PS lipids and a set of many smaller ones of $1-100$ PS lipids. This is clear from the snapshots shown in Figure 7. Since no attractive protein-protein interaction was incorporated into the model, it is clear that large protein and lipid clusters can be formed in a process mediated by preferential protein-lipid and lipid-lipid interactions in the membrane. We emphasize that both types of preferential interactions are required for protein-induced cluster formation

Variation of the E/M ratio as a function of protein concentration for a series of lipid mixtures with a PS content from 10 to $40 \%$ is mimicked in the computer simulations by the variation of domain size as a function of total added protein. Simulations resembling those obtained by varying PS from 10 to $40 \%$ at constant $\omega_{\text {ab }}(240 \mathrm{cal} / \mathrm{mol})$ can also be achieved by keeping the PS constant at $20 \%$ but varying the unlike lipid-lipid interaction parameter, $\omega_{\mathrm{ab}}$, between 200 and $320 \mathrm{cal} / \mathrm{mol}$ (compare Figure 5 with Figure 6, right). This underscores the sensitivity of the system to the magnitude of the unlike lipid-lipid interaction parameter. The changes in average domain size when $\omega_{\mathrm{ab}}$ is varied by about $100 \mathrm{cal} / \mathrm{mol}$ are so large that they resemble a variation of anionic lipid content from 10 to $40 \%$. It is important to note that these changes in the unlike lipid-lipid interaction parameter are extremely small (less that the thermal energy $k T$ ) but lead to substantial changes in domain sizes and their response to protein binding. This is particularly interesting because it suggests that the cluster sizes of both proteins and lipids are subject to fine-tuning based on lipid-lipid interactions in the membrane. Thus, proteins can show definite lipid preferences, which could be directly related to the specific lipid chemical structure and their tendencies to form specific types of domains (e.g., ordered or disordered, compact or elongated). It is also likely that interaction with the protein will modify the size and dynamics of lipid domain formation.

Membrane-associated proteins, the behavior of which is often modulated by specific lipids, mediate cellular signaling events. Slight perturbations in concentration of lipid and proteins, $\mathrm{pH}$, concentration of divalent cations, or electrostatic interactions may locally modify or even trigger component demixing and domain formation. For example, Mustonen and co-workers (40) used resonance energy transfer from pyrene-labeled lipids to cytochrome $c$ to monitor protein binding to lipid bilayers. Their results indicated that the extent of binding depended on phase separation in the bilayer and on ionic strength. They also suggested that membrane-bound cytochrome $c$ has a longrange ordering effect on the membrane lipids. Sorting of membrane components can thus be induced, imparting the capacity to locally assemble the machinery for a signal transduction or membrane trafficking event. It is our contention that the underlying lipid mixing properties are a means by which the behavior of membrane binding proteins can be finely regulated, with exquisite control, both temporally and spatially.

Since these clusters are dynamic, they will be continuously assembling and disassembling as components diffuse onto and off the membrane. Clusters could be composed of peripheral membrane binding proteins with similar lipid binding properties such as lipases (e.g., cytosolic phospholipase $\mathrm{A}_{2}$ and PI-specific phospholipase, PLC) and signaling proteins such as PKC, Src, Ras, PI3K, rasGAP, and unc 13. One might also postulate that some proteins that have no apparent enzymatic or signaling activities, like the annexins, might function specifically to organize lipid domains upon which other proteins can bind. The manner in which these putative clustered proteins are regulated is very different from the view of a 1/1 interaction between signal transduction machinery, mediated by a procession of phosphorylations producing a series of protein conformational changes activating one protein after another. Other ligands for some of the proteins mentioned above, such as diacylglycerol (DAG) and $\mathrm{Ca}^{2+}$, can also change the size, the shape, and the time scale of these domains. DAG and $\mathrm{Ca}^{2+}$ are potent perturbants of lipid mixing behavior and can readily modify the mixing properties of the lipid components, enhancing the tendency of a lipid mixture to form domains (6). DAG and $\mathrm{Ca}^{2+}$ would then be expected to also modify the membrane binding affinity of proteins that possess a preferential affinity for a specific lipid such as PS even in the absence of a specific protein binding site for DAG or $\mathrm{Ca}^{2+}$. If DAG and $\mathrm{Ca}^{2+}$ are also ligands, the observed modification in the binding of 
protein to lipid will be accentuated. Similarly, regulation of domain properties may be achieved via phosphorylation of the basic membrane binding surface of proteins, thus altering the magnitude of protein-lipid interactions.

The general concepts presented here regarding the coupling between the binding of proteins that exhibit preferential surface interactions and membrane domain formation apply as well to integral proteins. In the latter case, the coupling is the result of the segregation of integral membrane proteins into regions of the membrane with a bilayer thickness that most closely matches the transmembrane moiety of the protein, the so-called hydrophobic mismatch mechanism (41-45). These domains composed of integral membrane proteins and lipids may be further modulated by membrane curvature. Changes in membrane curvature have been shown to lead to differences between conformations of protein membrane spanning domains and altered ion channel formation (46). It has been suggested that coupling of membrane curvature with domain formation may also regulate membrane fusion or fission (47) or caveolae formation.

The biological membrane may also become structured as the neighborhood of integral membrane proteins becomes locally enriched in specific lipids, an enrichment that will stabilize a particular domain and can exhibit long-range lipidmediated organization. If there is a limited amount of the enriching lipid, it will be more favorable to cluster the proteins (48). Simulation of a conformational transition of an integral membrane protein has revealed that coupling of protein-lipid interactions to the conformational change (49) can restructure the surrounding lipid.

Lipid demixing could lead to formation of lipid rafts enriched in sphingolipid and cholesterol. Within the plasma membrane, PS is restricted to the inner leaflet (50). Sphingolipid and cholesterol rafts are found in the trans-Golgi network and the outer leaflet of the plasma membrane, among other sites. The unusually long lipids that predominate in rafts may strongly couple to the inner leaflet lipids and thereby, influence PS or other lipid-enriched domain formation on the inner leaflet of the plasma membrane. In addition, whether on the inner or outer portion of the plasma membrane, interaction of these protein-lipid complexes with the cytoskeleton or extracellular matrix can modify the tendency for domain formation.

It is clear that lipid-lipid and protein-lipid interactions can be thermodynamically coupled to produce dynamic heterogeneities in the biological membrane. These interactions can impart dynamic control on the spatial and temporal organization of signal transduction and trafficking machinery. Although many questions remain to be answered, the type of membrane organization described here could have important consequences in specific functions of membranes, and should be considered when seeking answers to questions of cellular function and regulation.

\section{ACKNOWLEDGMENT}

We thank Leah Shaw for helping with the experiments, Peter Holloway and Alfred Dumaual for helpful discussions, Dianne Murray for help in modeling of the protein, and Ed Hall of the Information Technology Center at the University of Virginia for help with graphical display of the snapshots. P.F.F.A. thanks the Universidade do Algarve, Portugal, for the kind permission to use their computational facilities and their continued support.

\section{REFERENCES}

1. Kinnunen, P. K. (1991) On the principals of functional ordering in biological membranes. Chem. Phys. Lipids 57, 375-399.

2. Bergelson, L. O., Gawrisch, K., Ferretti, J. A., and Blumenthal, R., Eds. (1995) Special issue on domain organization in biological membranes. Mol. Membr. Biol. 12, 1-162.

3. Vaz, W. L. C., and Almeida, P. F. F. (1993) Phase topology in multiphase lipid bilayers: is the biological membrane a domain mosaic? Curr. Opin. Struct. Biol. 3, 482-488.

4. Vaz, W. L. C., and Almeida, P. F. F. (2000) Lateral and transversal heterogeneity in membranes. in Biomembranes, Molecular Structure and Function, Chapter 4, Springer, New York (in press).

5. Hinderliter, A. K., Dibble, A. R. G., Biltonen, R., and Sando, J. J. (1997) Activation of protein kinase C by coexisting diacylglycerol-enriched and diacylglycerol-poor lipid domains. Biochemistry 36, 6141-6148.

6. Dibble, A. R. G., Hinderliter, A. K., Sando, J. J., and Biltonen, R. L. (1996) Lipid lateral heterogeneity in phosphatidylcholine/ phosphatidylserine/diacylglycerol vesicles and its influence on protein kinase C activation. Biophys. J. 71, 1877-1890.

7. Thompson, T. E., Sankaram, M. B., Biltonen, R. L., Marsh, D., and Vaz, W. L. C. (1995) Effects of domain structure on in-plane reactions and interactions. Mol. Membr. Biol. 12, $157-162$.

8. Melo, E. C., Lourtie, I. M., Sankaram, M. B., Thompson, T. E., and Vaz, W. L. C. (1992) Effects of domain connection and disconnection on the yields of in-plane bimolecular reactions in membranes. Biophys. J. 63, 1506-1512.

9. Rietveld, A., and Simons, K. (1998) The differential miscibility of lipids as the basis for the formation of functional membrane rafts. Biochim. Biophys. Acta 1376, 467-479.

10. Simons, K., and Ikonen, E. (1997) Functional rafts in cell membranes. Nature 387, 569-572.

11. Wu, H. S., and McConnell, H. M. (1975) Phase separations in phospholipid membranes. Biochemistry 14, 847-854.

12. Mabrey, S., and Sturtevant, J. (1976) Investigation of phase transitions of lipids and lipid mixtures by high sensitivity differential scanning calorimetry. Proc. Natl. Acad. Sci. U.S.A. 73, 3862-3866.

13. Knoll, W., Ibel, K., and Sackmann, E. (1981) Small-angle neutron scattering study of lipid phase diagrams by the contrast variation method. Biochemistry 20,6379-6383.

14. Korlach, J., Schwille, P., Webb, W. W., and Feigenson, G. W. (1999) Characterization of lipid bilayer phases by confocal microscopy and fluorescence correlation spectroscopy. Proc. Natl. Acad. Sci. U.S.A. 96, 8461-8466.

15. Sugár, I. P., Thompson, T. E., and Biltonen, R. L. (1999) Monte Carlo simulation of two-component bilayers: DMPC/ DSPC mixtures. Biophys. J. 76, 2099-2100.

16. Jørgensen, K., and Mouritsen, O. G. (1995) Phase separation dynamics and lateral organization of two-component lipid membranes. Biophys. J. 95, 942-954.

17. Jerala, R., Almeida, P. F. F., and Biltonen, R. L. (1996) Simulation of the gel-fluid transition in a membrane composed of lipids with two connected acyl chains: application of a dimer-move step. Biophys. J. 71, 609-615.

18. Ipsen, H. J., Karlström, G., Mouritsen, O. G., Wennerström, H., and Zuckermann, M. J. (1987) Phase equilibria in the phosphatidylcholine-cholesterol system. Biochim. Biophys. Acta 905, 162-172.

19. Sankaram, M. B., and Thompson, T. E. (1990) Modulation of phospholipid acyl chain order by cholesterol. A solid-state ${ }^{2} \mathrm{H}$ nuclear magnetic resonance study. Biochemistry 29, 1067610684.

20. Sankaram, M. B., and Thompson, T. E. (1991) Cholesterolinduced fluid-phase immiscibility in membranes. Proc. Natl. Acad. Sci. U.S.A. 88, 8686-8690.

21. Almeida, P. F. F., Vaz, W. L. C., and Thompson, T. E. (1992) Lateral diffusion in the liquid phases of dimyristoylphosphati- 
dylcholine/cholesterol lipid bilayers: a free volume analysis. Biochemistry 31, 6739-6747.

22. Ahmed, S. N., Brown, D. A., and London, E. (1997) On the origin of sphingolipid/cholesterol-rich detergent-insoluble cell membranes: physiological concentrations of cholesterol and sphingolipid induce formation of a detergent-insoluble, liquidordered lipid phase in model membranes. Biochemistry 36 , 10944-10953.

23. Von Dreele, P. H. (1978) Estimation of lateral species separation from phase transitions in nonideal two-dimensional lipid mixtures. Biochemistry 17, 3939-3943.

24. Sugár, I. P., Biltonen, R. L., and Mitchard, N. (1994) Monte Carlo simulation of membranes: phase transition of small unilamellar dipalmitoylphosphatidylcholine vesicles. Methods Enzymol. 240, 569-593.

25. Heimburg, T., and Biltonen, R. L. (1996) A Monte Carlo simulation study of protein-induced heat capacity changes and lipid-induced protein clustering. Biophys. J. 70, 84-96.

26. Kingsley, P. B., and Feigenson, G. W. (1979) The synthesis of a perdeuterated phospholipid: 1,2-dimyristoyl-sn-glycero3-phosphocholine- $d_{72}$. Chem. Phys. Lipids 24, 135-147.

27. Bradford, M. M. (1976) A rapid and sensitive method for the quantitation of microgram quantities of protein utilizing the principle of protein-dye binding. Anal. Biochem. 72, 248254.

28. Damer, C. K., and Creutz, C. E. (1996) Calcium-dependent self-association of synaptotagmin I. J. Neurochem. 67, 16611668.

29. Damer, C. K., and Creutz, C. E. (1994) Synergistic membrane interactions of the two $\mathrm{C} 2$ domains of synaptotagmin. J. Biol. Chem. 269, 31115-31123.

30. Binder, K., and Heermann, D. W. (1997) Monte Carlo simulation in statistical physics. Solid-State Sciences, 3rd ed., Springer, New York.

31. Macedo-Ribeiro, S., Bode, W., Huber, R., Quinn-Allen, M. A., Kim, W., Ortel, T. L., Bourenkov, G. P., Bertunik, H. D., Stubbs, M. T., Kane, W. H., and Fuentes-Prior, P. (1999) Crystal structures of the membrane-binding $\mathrm{C} 2$ domain of human coagulation factor V. Nature 402, 434-439.

32. Pratt, K. P., Shen, B. W., Tekeshima, K., Davie, E. W., Fujikawa, K., and Stoddard, B. L. (1999) Structure of the C2 domain of human factor VIII at $1.5 \AA$ resolution. Nature 402, 439-442.

33. Verdaguer, N., Corbalan-Garcia, S., Ochoa, W. F., Fite, I., and Gomez-Fernandez, J. C. (1999) $\mathrm{Ca}^{2+}$ bridges the C2 membrane-binding domain of protein kinase $\mathrm{Ca}$ directly to phosphatidylserine. EMBO J. 18, 6329-6338.

34. Metropolis, N., Rosenbluth, A. W., Rosenbluth, W. N., Teller, A. H., and Teller, E. (1953) Equation of state calculations by fast computing machines. J. Chem. Phys. 21, 1087-1092.

35. Junker, M., and Creutz, C. E. (1993) Endonexin (annexin IV)mediated lateral segregation of phosphatidylglycerol/phospatidylcholine membranes. Biochemistry 32, 9968-9974.

36. Hinderliter, A. K., Almeida, P. F. F., Biltonen, R. L., and Creutz, C. E. (1998) Membrane domain formation by calcium- dependent, lipid-binding proteins: insights from the $\mathrm{C} 2$ motif. Biochim. Biophys. Acta 1448, 227-235.

37. Denisov, G., Wanaski, S., Luan, P., Glaser, M., and McLaughlin, S. (1998) Binding of Basic Peptides to Membranes Produces Lateral Domains Enriched in the Acidic Lipids Phosphatidylserine and Phosphatidylinositol 4,5-Bisphosphate: An Electrostatic Model and Experimental Results. Biophys. J. 74, 731-744.

38. Gadd, M. E., and Biltonen, R. L. (2000) Characterization of the interaction of phospholipase $\mathrm{A}_{2}$ with phosphatidylcholinephosphatidylglycerol mixed lipids. Biochemistry 39, 96239631 .

39. Huang, J., Swanson, J. E., Dibble, A. R. G., Hinderliter, A. K., and Feigenson, G. W. (1993) Nonideal mixing of phosphatidylserine and phosphatidylcholine in the fluid lamellar phase. Biophys J. 64, 413-425.

40. Mustonen, P., Virtanen, J. A., Somerharju, P. J., and Kinnunen, P. K. J. (1987) Binding of cytochrome $c$ to liposomes as revealed by the quenching of fluorescene of pyrene-labeled phospholipids. Biochemistry 26, 2991-2997.

41. Mouritsen, O. G., and Bloom, M. (1984) Mattress model of lipid-protein interactions in membranes. Biophys. J. 46, 141153.

42. Bretscher, M. S., and Munro, S. (1993) Cholesterol and the Golgi apparatus. Science 261, 1280-1288.

43. Pelham, H., and Munro, S. (1993) Sorting of membrane proteins in the secretory pathway. Cell 75, 603-605.

44. Munro, S. (1995) An investigation of the role of transmembrane domains in Golgi protein retention. EMBO J. 14, 46954704.

45. Lehtonen, J. Y. A., and Kinnunen, P. K. J. (1997) Evidence for phospholipid microdomain formation in liquid crystalline liposomes reconstituted with Escherichia coli lactose permease. Biophys. J. 72, 1247-1257.

46. Lundbaek, J. A., Maer, A. M., and Anderson, O. S. (1997) Lipid bilayer electrostatic energy, curvature stress, and assembly of gramicidin channels. Biochemistry 36, 5695-5701.

47. Sackmann, E., and Feder, T. (1995) Budding, fission and domain formation in mixed lipid vesicles induced by phase separation and macromolecular condensation. Mol. Membr. Biol. 12, 21-29.

48. Gil, T., Ipsen, J. H., Mouritsen, O. G., Sabra, M. S., Sperotto, M. M., and Zuckermann, M. J. (1998) Theoretical analysis of protein organization in lipid membranes. Biochim. Biophys. Acta 1376, 245-266.

49. Sabra, M. C., and Mouritsen, O. G. (1998) Steady-state compartmentalization of lipid membranes by active proteins. Biophys. J. 74, 745-752.

50. Schroit, A. J., and Zwaal, R. F. A. (1991) Transbilayer movement of phospholipids in red cell and platelet membranes. Biochim. Biophys. Acta 1071, 313-329.

BI0024299 